\title{
Mortalidade por suicídio de adolescentes no Brasil: tendência temporal de crescimento entre 2000 e 2015
}

\author{
Suicide mortality among adolescents in Brazil: \\ increasing time trend between 2000 and 2015 \\ Júlia Isabel Richter Cicogna' \\ https://orcid.org/0000-0003-1489-110X \\ Danúbia Hillesheim² \\ https://orcid.org/0000-0003-0600-4072 \\ Ana Luiza de Lima Curi Halla2 \\ https://orcid.org/0000-0003-4761-0001
}

\section{RESUMO}

Objetivos: Analisar a tendência temporal de mortalidade por suicídio entre adolescentes no Brasil de 2000 a 2015. Métodos: Os dados foram coletados do Sistema de Informações sobre Mortalidade (SIM) e do Instituto Brasileiro de Geografia e Estatística (IBGE). As variáveis do estudo foram sexo, ano e causa básica de morte. O estudo incluiu mortes por Lesões Autoprovocadas Intencionalmente, X60-X84 - de acordo com a 10a Revisão da Classificação Internacional de Doenças (CID-10), de adolescentes de 10 a 19 anos. A técnica estatística utilizada foi a de regressão linear simples, e os resultados foram considerados estatisticamente significativos quando $p \leq 5 \%$. Resultados: Entre 2000 e 2015, foram observados 11.947 óbitos por suicídio em adolescentes no Brasil, e $67 \%$ deles foram no sexo masculino, o que corresponde a uma relação de 2,06:1 entre o sexo. Houve crescimento estatisticamente significativo da mortalidade por suicídio de adolescentes no Brasil $(p=0,016)$, a qual passou de 1,71 por 100.000 habitantes em 2000 para 2,51 em 2015, um crescimento de 47\%. Essa expansão ocorreu em função do incremento nos suicídios de adolescentes do sexo masculino ( $p=0,001)$, especificamente no norte $(p<0,001)$ e nordeste $(p<0,001)$ do Brasil. Em relação ao sexo feminino, houve tendência de diminuição da mortalidade por suicídio na região

\section{Palavras-chave}

Adolescente, suicídio, análise de regressão, Brasil. Centro-Oeste $(p=0,039)$, mas, quando se trata do Brasil como um todo, houve oscilação da mortalidade por suicídio. Conclusões: Houve crescimento significativo da mortalidade por suicídio entre os adolescentes no Brasil, particularmente na população masculina.

\begin{abstract}
Objectives: Analyze the suicide mortality time trends among adolescents in Brazil from 2000 to 2015. Methods: Data were collected from the Brazilian Mortality Database and from the Brazilian Institute of Geography and Statistics. Study variables were sex, year and underlying cause of death. The study included deaths from Intentional Self-Harm, X60-X84 - according to the 10th Revision of the International Classification of Diseases (ICD-10), of adolescents aged 10 to 19. The simple linear regression technique was used and results were considered
\end{abstract}

1 Universidade Federal de Santa Catarina (UFSC), Curso de Medicina, Florianópolis, SC, Brasil. 2 Universidade Federal de Santa Catarina (UFSC), Florianópolis, SC, Brasil.

Endereço para correspondência: Júlia Isabel Richter Cicogna Av. Me. Benvenuta 411, Bloco E, ap. 202 88040-900 - Florianópolis, SC, Brasil E-mail: jrichtercicogna@hotmail.com 


\section{Keywords}

Adolescent, suicide, regression analysis, Brazil. statistically significant when $p \leq 5 \%$. Results: From 2000 to 2015, there were 11,947 deaths due to suicide of adolescents in Brazil and $67 \%$ of these occurred in male adolescents, which corresponds to a 2,06:1 male-female ratio. There was a statistically significant increase in adolescent suicide mortality in Brazil $(p=0.016)$, which increased from 1.71 per 100,000 inhabitants in 2000 to 2.51 in 2015, a raise of $47 \%$. The increase occurred in behalf of the increment in suicides of male adolescents $(p=0.001)$ specifically in the North $(p<0.001)$ and Northeast $(p<0.001)$ of Brazil. In regard to the female group, there was a downtrend of mortality by suicide in the Center West region ( $p=0.039$ ), but when it comes to Brazil as a whole, there was a stabilization behavior of mortality by suicide. Conclusions: These results indicate an increase in the suicide rate of adolescents in Brazil, particularly in the male population. The improvement of suicide prevention strategies in Brazil is imperative.

\section{INTRODUÇÃO}

O suicídio é um fenômeno complexo, de impacto tanto individual quanto coletivo, que ocorre por uma convergência entre fatores de risco genéticos, psicológicos, sociais e culturais, combinados com experiências de trauma e perda'.

A cada ano, cerca de 800.000 mortes por suicídio ocorrem no mundo, o que representa uma morte a cada 40 segundos. Entre os jovens (15 a 29 anos), é a segunda causa de morte globalmente, segundo estimativas da Organização Mundial da Saúde $(\mathrm{OMS})^{2}$. As maiores taxas anuais de suicídio ocorrem no Leste Europeu, onde 10 países reportaram mais de 27 suicídios por 100.000 pessoas; já a América Latina e os países muçulmanos têm as menores taxas, menos de 6,5 por $100.000^{3}$. Entretanto, é questionado se os dados são subestimados, uma vez que valores culturais e sociais, com maior ou menor impacto dependendo da região, podem afetar o procedimento de registro da morte por lesão autoprovocada intencionalmente ${ }^{4}$.

Estatísticas em relação ao suicídio variam conforme localizações geográficas, sexo e faixas etárias. Em consonância com outros países, as taxas de suicídio no Brasil têm aumentado, passando de 4,4 a cada 100.000 habitantes em 1980 para 5,8 em 2006. Isso ocorre à custa do incremento nas taxas do fenômeno na população masculina. Registraram-se quatro óbitos masculinos para cada óbito feminino por suicídio em 1980 e 5,4:1 em 20065. No Brasil, as variações regionais são expressivas, e em algumas localidades, como o interior do Rio Grande do Sul, os índices se assemelham aos dos países do Leste Europeú.

A adolescência é um período de transição em que o indivíduo é especialmente vulnerável a reagir com atitude suicida em resposta a conflitos. História de adoção, homossexualidade, bissexualidade ou o questionamento da orientação sexual, história de abuso sexual, depressão e outros transtornos psiquiátricos, estresse pós-traumático, abuso de substâncias e uso patológico de internet estão entre fatores de risco para o suicídio entre adolescentes. Além disso, adolescentes são mais influenciáveis por exposição midiática do que adultos e podem imitar comportamentos vistos na televisão, com potencial para epidemias de suicídio quando o assunto é exposto?.

O suicídio é incomum antes dos 15 anos de idade, porém a sua prevalência aumenta na adolescência. No mundo, adolescentes do sexo masculino têm maior probabilidade de morrer por suicídio do que as adolescentes do sexo feminino, sendo a taxa entre os meninos de 15 a 19 anos duas a seis vezes mais alta do que a taxa em meninas da mesma idade ${ }^{8}$. O suicídio, no entanto, não é menos importante entre a população feminina, representando a principal causa de morte entre meninas de 15 a 19 anos e a terceira em meninos na mesma faixa etária9.

Apesar de existirem estudos que investigaram a mortalidade por suicídio entre adolescentes em localizações específicas no Brasil, não se pode extrapolar esses dados para a descrição do país, considerando a variação regional. Assim, tendo em vista a importância do suicídio como causa de morte entre adolescentes e a variação temporal, regional e de gênero que pode ocorrer entre as taxas desse evento, este estudo tem por objetivo analisar a tendência temporal de mortalidade por suicídio em adolescentes no Brasil, segundo macrorregiões e sexo, no período de 2000 a 2015.

\section{MÉTODOS}

Trata-se de um estudo descritivo de tendência temporal da taxa de mortalidade por suicídio de adolescentes no Brasil, segundo macrorregiões, de 2000 a 2015.

Os dados foram obtidos do Sistema de Informações sobre Mortalidade (SIM) e as informações relativas à população residente do Brasil e suas macrorregiões foram obtidas do censo populacional de $2010^{10}$ e das estimativas populacionais do Instituto Brasileiro de Geografia e Estatística (IBGE) para os demais anos.

Foram considerados suicídio os óbitos por lesões autoprovocadas intencionalmente, codificadas como X60 a X84, segundo a Classificação Internacional de Doenças, 10a Revisão (CID-10) ${ }^{11}$. Os óbitos foram estudados segundo a variável sexo. Foram incluídos no estudo indivíduos de 10 a 19 anos. 
Para descrição e análise dos dados, foram obtidos, para cada ano do calendário, os coeficientes médios brutos de óbitos por lesões autoprovocadas intencionalmente, segundo macrorregião, em cada sexo. As taxas foram calculadas para cada 100.000 habitantes.

Foi realizada a análise de tendência das taxas de mortalidade, para o Brasil e suas macrorregiões, utilizando-se a técnica estatística de regressão linear simples, por meio do software Stata 14. Justifica-se a opção por essa modelagem pela facilidade de elaboração, interpretação e poder estatístico. Foram considerados, como variável dependente (Y), os coeficientes padronizados de mortalidade por suicídio e, como variável independente $(X)$, os anos-calendário do estudo. $O$ modelo linear foi definido como $y=\beta 0+\beta 1 x$, sendo $\beta 0$ o coeficiente médio do período e $\beta 1$ o incremento (acréscimo ou decréscimo) médio do período (variação média anual). Admitiu-se tendência linear estatisticamente significante somente quando a sua probabilidade de ter ocorrido foi igual ou menor do que 0,05 , ou seja, $\mathrm{p} \leq 5 \%$.

\section{RESULTADOS}

De 2000 a 2015, no Brasil, ocorreram 11.947 mortes por lesões autoprovocadas intencionalmente em indivíduos de 10 a 19 anos. Isso representa 8,25\% do total de óbitos por suicídio em todas as faixas etárias no período. A maior parte (85,32\%) dos suicídios na faixa etária estudada aconteceu em adolescentes de 15 a 19 anos. A maioria dos óbitos por suicídio em adolescentes no Brasil no período ocorreu na população masculina $(67,31 \%)$ e a proporção da mortalidade entre a população masculina e a feminina foi de 2,06:1 em todo o período (Tabela 1).

O principal método de suicídio utilizado nessa faixa etária em ambos os sexos no período foi o enforcamento, estrangulamento ou sufocação (X70), o qual foi responsável por $58,95 \%$ das mortes. O segundo método mais frequentemente utilizado foi o disparo de arma de fogo não especificada (X74), correspondendo a 9,75\% das mortes. Em seguida, aparece a autointoxicação a pesticidas (X68), com 7,99\%.

O coeficiente de mortalidade por lesões autoprovocadas intencionalmente na faixa etária de 10 a 19 anos em ambos os sexos no Brasil em 2000 foi de 1,71 óbito a cada 100.000 habitantes. Em 2015, o coeficiente foi de 2,51. Esses resultados representam crescimento de $47 \%$ na mortalidade por suicídio em adolescentes no Brasil no período estudado (Tabela 2).

Considerando ambos os sexos, na região Norte o coeficiente de mortalidade por suicídio teve crescimento de $72,81 \%$, tendo variado de 2,28 a cada 100.000 habitantes em 2000 para 3,94 em 2015. Na região Nordeste, o coeficiente passou de 1,14 a cada 100.000 habitantes em 2000 para 2,14 em 2015, o que representou um aumento de $87,72 \%$. A região Centro-Oeste revelou os maiores coeficientes de mortalidade por suicídio de adolescentes no Brasil. A região Sul foi a segunda região com mais mortes por suicídio no período (Tabela 2).

Tabela 1. Distribuição do número e percentual de óbitos por lesões autoprovocadas intencionalmente no Brasil, na faixa etária de 10 a 19 anos, segundo ano de óbito e sexo - Proporção da mortalidade na população masculina pela feminina - Brasil, 2000 a 2015

\begin{tabular}{|c|c|c|c|c|c|c|}
\hline Ano do Óbito & Masculino & $\%$ & Feminino & $\%$ & Total & Masculino: Feminino \\
\hline 2000 & 384 & 63,16 & 224 & 36,84 & 608 & $1,71: 1$ \\
\hline 2001 & 533 & 65,64 & 279 & 34,36 & 812 & $1,91: 1$ \\
\hline 2002 & 493 & 65,47 & 260 & 34,53 & 753 & $1,90: 1$ \\
\hline 2003 & 500 & 66,05 & 257 & 33,95 & 757 & $1,95: 1$ \\
\hline 2004 & 482 & 64,87 & 261 & 35,13 & 743 & 1,85:1 \\
\hline 2005 & 468 & 64,29 & 260 & 35,71 & 728 & $1,80: 1$ \\
\hline 2006 & 496 & 66,31 & 252 & 33,69 & 748 & $1,97: 1$ \\
\hline 2007 & 484 & 67,79 & 230 & 32,21 & 714 & $2,10: 1$ \\
\hline 2008 & 480 & 65,93 & 248 & 34,07 & 728 & $1,94: 1$ \\
\hline 2009 & 483 & 71,88 & 189 & 28,13 & 672 & $2,56: 1$ \\
\hline 2010 & 489 & 69,26 & 217 & 30,74 & 706 & $2,25: 1$ \\
\hline 2011 & 487 & 66,44 & 246 & 33,56 & 733 & $1,98: 1$ \\
\hline 2012 & 554 & 69,95 & 238 & 30,05 & 792 & $2,33: 1$ \\
\hline 2013 & 555 & 70,70 & 230 & 29,30 & 785 & $2,41: 1$ \\
\hline 2014 & 561 & 68,92 & 253 & 31,08 & 814 & $2,22: 1$ \\
\hline 2015 & 593 & 69,44 & 261 & 30,56 & 854 & $2,27: 1$ \\
\hline Total & 8042 & 67,31 & 3905 & 32,69 & 11947 & $2,06: 1$ \\
\hline
\end{tabular}

Fonte: Sistema de Informaçōes sobre Mortalidade (SIM). 
Entre os meninos, o coeficiente de mortalidade por suicídio foi de 2,12 em 2000 e de 3,42 em 2015, o que representou um aumento de $61,15 \%$. Na região Norte, o coeficiente de mortalidade era de 3,03 em 2000 e de 5,06 em 2015, o que representou um aumento de $67,15 \%$. Na região Nordeste, o aumento foi de 141,44\%; em 2000 o coeficiente era de 1,17 e em 2015 passou para 2,83. Da mesma maneira que nos dados de ambos os sexos, a região com maiores coeficientes de mortalidade na população masculina foi a Centro-Oeste (Tabela 2).

Entre as meninas, o coeficiente de mortalidade por suicídio no Brasil foi de 1,28 em 2000 e de 1,56 em 2015. A região Centro-Oeste revelou-se aquela em que ocorreram mais suicídios de adolescentes do sexo feminino no período (Tabela 2).

Na figura 1 são apresentados os coeficientes de mortalidade por suicídio em adolescentes em ambos os sexos no Brasil, segundo os anos estudados. Observa-se tendência de crescimento no Brasil e nas regiões Norte e Nordeste (Figura 1).

Houve tendência de aumento na mortalidade por suicídio em adolescentes em ambos os sexos no Brasil no período estudado $(p=0,01)$, com incremento médio de 0,02 óbito a cada ano. Observou-se, ainda, tendência de aumento entre os adolescentes do sexo masculino no Brasil $(p=0,00)$, enquanto no sexo feminino ocorreu flutuação nos valores, não tendo sido estatisticamente significante. A região Norte apresentou crescimento, considerando o sexo masculino e ambos os sexos $(p<0,001)$. A região Nordeste seguiu a mesma tendência, com incremento de 0,08 óbito ao ano na população masculina ( $p<0,001)$ e de 0,04 em ambos os sexos $(p<0,001)$. Nas regiões Sul e Sudeste houve flutuação das taxas. A região Centro-Oeste revelou uma tendência de diminuição dos óbitos por suicídio na população feminina $(p=0,03)$ (Tabela 3).

\section{DISCUSSÃO}

O presente estudo observou tendência de crescimento das taxas de óbitos de adolescentes de 10 a 19 anos por suicídio no Brasil, de 2000 a 2015. Isso ocorreu à custa do incremento na taxa de óbitos masculino nas regiões Norte e Nordeste. Entre a população feminina, houve tendência de diminuição na região Centro-Oeste, porém, quando se avaliou o Brasil como um todo, ocorreu oscilação nas taxas de óbitos por lesões autoprovocadas intencionalmente no período. A região Centro-Oeste, apesar de não ter apresentado tendência de aumento, revelou-se a região com maior taxa de óbitos por suicídio.

Tabela 2. Coeficiente de mortalidade por lesões autoprovocadas intencionalmente de adolescentes de 10 a 19 anos, segundo sexo, macrorregiões brasileiras e o ano do óbito - Brasil, 2000 a 2015

\begin{tabular}{|c|c|c|c|c|c|c|c|c|c|c|c|c|c|c|c|c|}
\hline \multicolumn{17}{|c|}{ Ano } \\
\hline Região & 2000 & 2001 & 2002 & 2003 & 2004 & 2005 & 2006 & 2007 & 2008 & 2009 & 2010 & 2011 & 2012 & 2013 & 2014 & 2015 \\
\hline \multicolumn{17}{|l|}{ Norte } \\
\hline Masculino & 3,03 & 3,37 & 3,09 & 3,25 & 3,17 & 2,84 & 3,11 & 4,67 & 4,27 & 5,04 & 4,43 & 3,82 & 4,35 & 5,28 & 5,19 & 5,06 \\
\hline Feminino & 1,50 & 2,39 & 1,99 & 1,85 & 1,64 & 2,13 & 1,55 & 1,35 & 1,88 & 1,69 & 1,85 & 2,43 & 1,64 & 2,33 & 2,21 & 2,78 \\
\hline Ambos & 2,28 & 2,89 & 2,55 & 2,56 & 2,42 & 2,49 & 2,34 & 3,04 & 3,10 & 3,39 & 3,17 & 3,14 & 3,02 & 3,83 & 3,73 & 3,94 \\
\hline \multicolumn{17}{|l|}{ Nordeste } \\
\hline Masculino & 1,17 & 1,85 & 2,14 & 1,69 & 1,94 & 2,11 & 2,03 & 2,52 & 2,26 & 2,31 & 2,49 & 2,21 & 2,61 & 2,71 & 2,83 & 2,83 \\
\hline Feminino & 1,10 & 1,07 & 1,01 & 1,58 & 1,46 & 1,39 & 1,65 & 1,42 & 1,74 & 1,10 & 1,42 & 1,52 & 1,26 & 1,29 & 1,31 & 1,43 \\
\hline Ambos & 1,14 & 1,47 & 1,58 & 1,64 & 1,70 & 1,75 & 1,85 & 1,98 & 2,00 & 1,71 & 1,96 & 1,87 & 1,95 & 2,01 & 2,08 & 2,14 \\
\hline \multicolumn{17}{|l|}{ Sudeste } \\
\hline Masculino & 1,44 & 2,75 & 2,17 & 2,40 & 2,16 & 1,91 & 2,49 & 2,02 & 1,75 & 1,66 & 1,95 & 2,27 & 2,39 & 2,18 & 2,10 & 2,47 \\
\hline Feminino & 0,83 & 1,34 & 1,19 & 1,08 & 0,96 & 0,98 & 0,97 & 1,00 & 1,05 & 0,67 & 0,98 & 1,20 & 1,13 & 0,86 & 1,14 & 1,18 \\
\hline Ambos & 1,14 & 2,06 & 1,69 & 1,75 & 1,57 & 1,45 & 1,74 & 1,52 & 1,40 & 1,17 & 1,47 & 1,74 & 1,77 & 1,53 & 1,63 & 1,83 \\
\hline \multicolumn{17}{|l|}{ Sul } \\
\hline Masculino & 4,17 & 5,22 & 4,59 & 5,06 & 4,72 & 4,61 & 4,29 & 3,14 & 4,23 & 4,28 & 3,68 & 4,16 & 4,31 & 4,56 & 4,95 & 4,83 \\
\hline Feminino & 1,81 & 2,36 & 2,36 & 1,44 & 2,09 & 1,75 & 1,97 & 2,02 & 1,68 & 1,12 & 1,44 & 1,09 & 1,76 & 1,73 & 2,24 & 1,27 \\
\hline Ambos & 3,01 & 3,82 & 3,50 & 3,29 & 3,43 & 3,21 & 3,16 & 2,59 & 2,98 & 2,73 & 2,58 & 2,66 & 3,06 & 3,17 & 3,62 & 3,09 \\
\hline \multicolumn{17}{|l|}{$\begin{array}{l}\text { Centro- } \\
\text { Oeste }\end{array}$} \\
\hline Masculino & 5,19 & 4,12 & 4,67 & 4,97 & 4,80 & 5,09 & 4,83 & 4,48 & 5,23 & 4,58 & 4,72 & 3,92 & 5,80 & 4,94 & 4,86 & 5,92 \\
\hline Feminino & 3,33 & 2,90 & 3,06 & $2,973,98$ & 3,61 & 3,84 & 2,52 & 1,78 & 1,60 & 2,79 & 1,27 & 1,90 & 2,52 & 2,36 & 1,97 & 2,91 \\
\hline Ambos & 4,27 & 3,52 & 3,87 & & 4,21 & 4,48 & 3,69 & 3,15 & 3,44 & 3,70 & 3,02 & 2,92 & 4,19 & 3,67 & 3,44 & 4,45 \\
\hline \multicolumn{17}{|l|}{ Brasil } \\
\hline Masculino & 2,12 & 2,96 & 2,75 & 2,81 & 2,72 & 2,66 & 2,83 & 2,76 & 2,74 & 2,76 & 2,80 & 2,79 & 3,17 & 3,18 & 3,22 & 3,42 \\
\hline Feminino & 1,28 & 1,60 & 1,49 & 1,49 & 1,52 & 1,52 & 1,48 & 1,36 & 1,46 & 1,12 & 1,28 & 1,46 & 1,41 & 1,37 & 1,51 & 1,56 \\
\hline Ambos & 1,71 & 2,29 & 2,13 & 2,16 & 2,13 & 2,10 & 2,16 & 2,07 & 2,11 & 1,95 & 2,05 & 2,13 & 2,31 & 2,29 & 2,38 & 2,51 \\
\hline
\end{tabular}

Fonte: Sistema de Informações sobre Mortalidade (SIM); Censo populacional de 2010 do Instituto Brasileiro de Geografia e Estatística (IBGE). 


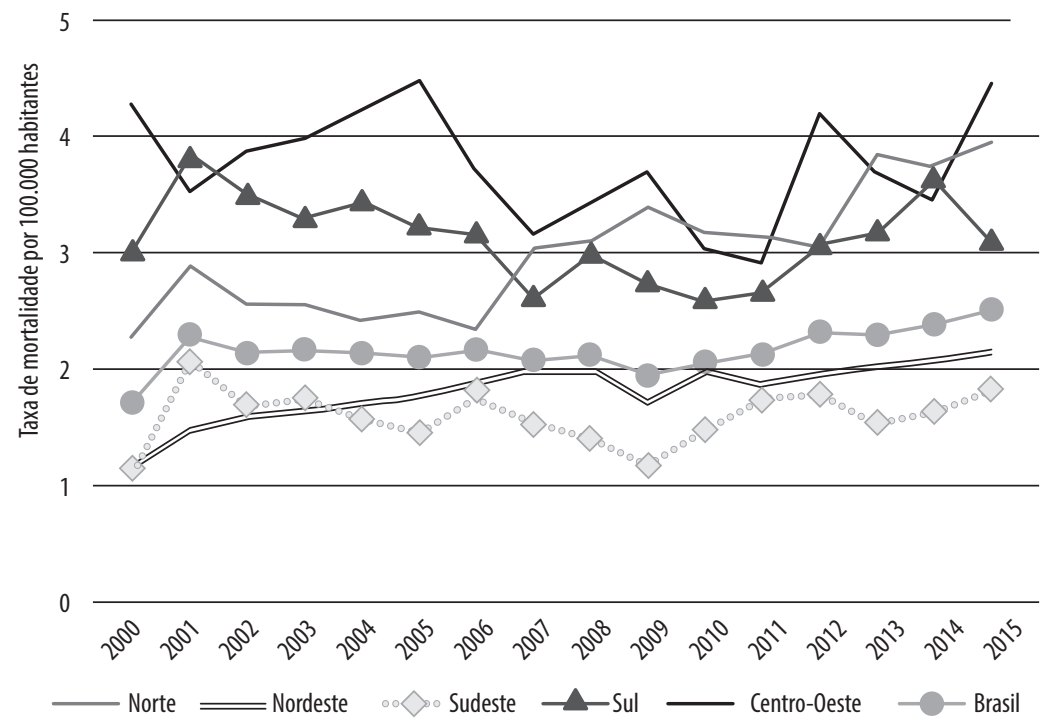

Figura 1. Tendência anual dos coeficientes de mortalidade por lesões autoprovocadas intencionalmente de adolescentes de 10 a 19 anos de ambos os sexos no Brasil, segundo macrorregiões, em 2000-2015.

Fonte: Sistema de Informações sobre Mortalidade (SIM); Censo populacional de 2010 do Instituto Brasileiro de Geografia e Estatística (IBGE).

Tabela 3. Tendência anual dos coeficientes de mortalidade por lesões autoprovocadas intencionalmente de adolescentes de 10 a 19 anos, segundo sexo e macrorregiões do Brasil - 2000 a 2015

\begin{tabular}{|c|c|c|c|c|}
\hline Macrorregiöes & Variação média anual (IC 95\%*) & Tendência & Valorp & $R^{2}$ \\
\hline \multicolumn{5}{|l|}{ Norte } \\
\hline Masculino & $0,15(0,09 ; 0,21)$ & Aumento & $<0,001$ & 0,70 \\
\hline Feminino & $0,03(-0,00 ; 0,07)$ & Estável & 0,106 & 0,17 \\
\hline Ambos & $0,09(0,06 ; 0,12)$ & Aumento & $<0,001$ & 0,74 \\
\hline \multicolumn{5}{|l|}{ Nordeste } \\
\hline Masculino & $0,08(0,05 ; 0,10)$ & Aumento & $<0,001$ & 0,79 \\
\hline Feminino & $0,01(-0,01 ; 0,03)$ & Estável & 0,358 & 0,06 \\
\hline Ambos & $0,04(0,03 ; 0,06)$ & Aumento & $<0,001$ & 0,76 \\
\hline \multicolumn{5}{|l|}{ Sul } \\
\hline Masculino & $-0,01(-0,07 ; 0,05)$ & Estável & 0,685 & 0,01 \\
\hline Feminino & $-0,03(-0,08 ; 0,00)$ & Estável & 0,094 & 0,18 \\
\hline Ambos & $-0,02(-0,06 ; 0,01)$ & Estável & 0,231 & 0,10 \\
\hline \multicolumn{5}{|l|}{ Sudeste } \\
\hline Masculino & $0,00(-0,03 ; 0,04)$ & Estável & 0,691 & 0,01 \\
\hline Feminino & $0,00(-0,01 ; 0,02)$ & Estável & 0,963 & 0,00 \\
\hline Ambos & $0,00(-0,02 ; 0,03)$ & Estável & 0,775 & 0,00 \\
\hline \multicolumn{5}{|l|}{ Centro-0este } \\
\hline Masculino & $0,03(-0,02 ; 0,09)$ & Estável & 0,242 & 0,09 \\
\hline Feminino & $-0,07(-0,15 ;-0,04)$ & Diminuiç̧ão & 0,039 & 0,26 \\
\hline Ambos & $-0,02(-0,07 ; 0,03)$ & Estável & 0,436 & 0,04 \\
\hline \multicolumn{5}{|l|}{ Brasil } \\
\hline Masculino & $0,04(0,02 ; 0,07)$ & Aumento & 0,001 & 0,56 \\
\hline Feminino & $-0,00(-0,01 ; 0,01)$ & Estável & 0,737 & 0,00 \\
\hline Ambos & $0,02(0,00 ; 0,40)$ & Aumento & 0,016 & 0,34 \\
\hline
\end{tabular}

* Intervalo de confiança de $95 \%$

Fonte: Sistema de Informaçōes sobre Mortalidade (SIM); Censo populacional de 2010 do Instituto Brasileiro de Geografia e Estatística (IBGE). 
Segundo o Mapa da Violência, a participação do suicídio no total de óbitos de crianças e adolescentes aumentou de 0,2\% em 1980 para 1\% em 2013, no Brasil. Nesse período, foi a segunda causa que mais cresceu, atrás apenas dos homicídios $^{12}$. Abasse et al. concluíram em seu estudo que, apesar de a mortalidade por suicídio em adolescentes ter sido considerada baixa em Minas Gerais, menor que 5 para 100.000 habitantes, a faixa etária de 10 a 19 anos representou 16,2\% do total das internações no Sistema Único de Saúde (SUS) decorrentes de tentativas de suicídio ${ }^{13}$. Borges et al. identificaram ideação suicida em 31,9\% de adolescentes da cidade de Erechim, o que é especialmente preocupante quando se considera que foi investigada uma população não clínica' ${ }^{14}$. Ademais, no "Boletim epidemiológico de tentativas e óbitos por suicídio", publicado pela Secretaria de Vigilância do Ministério da Saúde, em setembro de 2017, foi observado que 44,8\% dos suicídios na população indígena foram cometidos por adolescentes (10 a 19 anos) $)^{15}$. Em conjunto com o efetivo aumento na mortalidade por suicídio em adolescentes encontrado neste trabalho, esses dados tornam clara a importância do suicídio como causa de morte de adolescentes no Brasil.

O coeficiente de mortalidade observado no presente estudo, considerando ambos os sexos, foi de 2,51 a cada 100.000 habitantes. Segundo dados de 2016 da OMS, no Brasil, em todas as idades, esse coeficiente foi de 6,5 a cada $100.000^{16}$. Há na literatura relatos de aumento da ocorrência de suicídios entre a população brasileira em geral17-19, o que respalda a tendência de aumento encontrada na mortalidade por suicídio de adolescentes. Da mesma maneira do que é destacado aqui para os adolescentes, o Nordeste foi apontado como a região com maior aumento na taxa de suicídios em um estudo realizado com adultos ${ }^{17}$. O crescimento do número de suicídios em crianças e adolescentes na região Norte também foi identificado em estudo realizado no Pará20.

O Centro-Oeste revelou-se a região brasileira com mais mortes por suicídios em adolescentes. Isso corresponde aos resultados de outros estudos, que também apontaram esse estado como a liderança nacional em suicídios, tanto em adultos como em jovens e adolescentes ${ }^{21,22}$. Machado e Santos analisaram a incidência de suicídios na população com mais de 10 anos no Brasil, entre 2000 e 2012, e identificaram maior mortalidade na região Sul, seguida da região Centro-Oeste ${ }^{17}$. O presente estudo também observou maior mortalidade nessas duas regiões, tendo, porém, a liderança do Centro-Oeste.

Conforme foi demonstrado no artigo, a razão entre a taxa de mortalidade da população adolescente masculina pela feminina foi de 2,06:1 em todo o período, o que vai ao encontro dos achados anteriores de maior taxa de suicídio entre homens, tanto no Brasil quanto no mundo 4,5,17. Entre a população brasileira adulta, a razão era de 3,60:1 em $2012^{17}$.

Kõlves e De Leo analisaram o suicídio entre adolescente de 15 a 19 anos em 81 países, de 1990 a 2009. Os autores verificaram que as taxas de suicídio em adolescentes do sexo masculino estão diminuindo entre os continentes, com exceção da América do Sul. Essa região, em oposição às outras regiões do mundo, apresentou aumento significativo das mortes de adolescentes por suicídio no período analisado, corroborando os resultados deste estudo ${ }^{23}$. Tendo em vista esses dados, percebe-se que o Brasil, com os seus países vizinhos, apresenta tendência oposta à mundial em relação ao suicídio de adolescentes. Isso reafirma a relevância do suicídio como causa de óbito de adolescentes no Brasil.

Os dados expostos aqui tornam clara a necessidade de que estratégias para a prevenção de suicídio de adolescentes sejam implementadas. As estratégias de prevenção de suicídio, segundo um estudo realizado na cidade de Pelotas, devem priorizar adolescentes do sexo feminino, principalmente as que são sexualmente ativas e apresentam sintomas de distúrbios da saúde mental e de abuso de substâncias ${ }^{24}$. No entanto, conforme foi elucidado no presente estudo, há substanciais variações regionais em relação ao suicídio, e assim a prevenção deve levar em consideração os fatores de risco de cada região.

Destaca-se que entre as limitações do presente estudo está a subnotificação ${ }^{25}$. Um estudo realizado em Recife observou que, dos casos de morte de adolescentes inicialmente classificados como sendo de intencionalidade indeterminada de 2000 a 2014, 9\% foram reclassificados como suicídio. Isso representou um percentual geral de 23,7\% de subnotificação ${ }^{26}$. Outra limitação diz respeito à cobertura e à qualidade do SIM, que, embora tenha melhorado substancialmente ao longo dos anos, ainda apresenta uma subenumeração de óbitos captados, especialmente nas regiões Norte e Nordeste ${ }^{27}$. Não se pode excluir que a melhora na abrangência do SIM, principalmente nas regiões Norte e Nordeste, tenha interferido nos dados coletados, provocando aumento das notificações, e não do número real de suicídios.

Por outro lado, considerando essa melhoria na qualidade e abrangência do SIM, este artigo possui como potencialidade ter usado uma base de dados de alcance nacional, o que permite traçar um panorama brasileiro referente aos suicídios. Segundo publicação da OMS, o Brasil figura entre o restrito grupo de 60 países que têm dados vitais de boa qualidade e que podem ser usados diretamente para estimar as taxas de suicídio². Além disso, o presente estudo contribui com a Portaria no 1.876 28 , a qual instituiu em 2006 as diretrizes nacionais para a prevenção do suicídio, no que se refere a um de seus objetivos, que é a coleta e a análise de dados para que se permita a qualificação da gestão e a disseminação de informações e conhecimentos em relação aos suicídios. Por fim, não foram encontrados dados recentes na literatura que investiguem mortes por lesões autoprovocadas intencionalmente entre a população adolescente (10 a 19 anos) no Brasil como um todo, o que potencializa a relevância do artigo. 


\section{CONCLUSÕES}

Os dados apresentados neste estudo indicam que a mortalidade de adolescentes por suicídio no Brasil apresentou tendência de crescimento nos últimos anos, especialmente entre a população masculina das regiões norte e nordeste no Brasil. Essas informações sugerem que os esforços nacionais para a prevenção de suicídio devem ser expandidos. Para que se aperfeiçoem as estratégias de prevenção ao suicídio, mais informações em relação aos fatores de risco específicas para adolescentes de cada macrorregião devem ser desenvolvidas. Por fim, destaca-se a importância de que profissionais da saúde, em especial os que trabalham nas regiões mais afetadas, estejam informados sobre esses dados, para que possam diagnosticar e intervir mais cedo na prevenção do suicídio em adolescentes.

\section{CONTRIBUIÇÕES INDIVIDUAIS}

Júlia I. R. Cicogna - Contribuiu com a concepção do estudo, interpretação dos dados, redação e revisão do artigo final.

Danúbia Hillesheim - Contribuiu com a análise dos dados, redação e revisão do artigo.

Ana Luiza de Lima Curi Hallal - Contribuiu com a concepção e delineamento do estudo, redação, normatização e revisão final do artigo.

Júlia I. R. Cicogna, Danúbia Hillesheim e Ana Luiza de Lima Curi Hallal, autoras do manuscrito, declaram que o trabalho descrito não foi publicado anteriormente e que não está em consideração para publicação em outro lugar. Todas as autoras aprovaram a versão final a ser publicada.

\section{CONFLITOS DE INTERESSE}

As autoras declaram não haver conflitos de interesse.

\section{REFERÊNCIAS}

1. Zalsman G, Hawton K, Wasserman D, van Heeringen K, Arensman E, Sarchiapone M, et al. Suicide prevention strategies revisited: 10-year systematic review. Lancet Psychiatry. 2016;3(7):646-59.

2. World Health Organization. Preventing suicide. CMAJ. 2014;143(7):609-10.

3. Mann JJ, Apter A, Bertolote J, Beautrais A, Currier D, Haas A, et al. Suicide prevention strategies: a systematic review. JAMA. 2005;294(16):2064-74.

4. Hawton K, van Heeringen K. Suicide. Lancet. 2009:373(9672):1372-81.

5. Minayo MCDS, Pinto LW, Assis SG, Cavalcante FG, Mangas RMDN. Tendência da mortalidade por suicídio na população brasileira e idosa, 1980-2006. Rev Saúde Pública. 2012;46(2):300-9.

6. Botega NJ. Comportamento suicida: epidemiologia. Psicol USP. 2014;25(3):231-6.
7. Shain B; Committee on Adolescence. Suicide and Suicide Attempts in Adolescents. Pediatrics. 2016;138(1)

8. Hawton K, Saunders KEA, O'Connor RC. Self-harm and suicide in adolescents. Lancet. 2012;379(9834):2373-82.

9. Patton GC, Coffey C, Sawyer SM, Viner RM, Haller DM, Bose K, et al. Global patterns of mortality in young people: a systematic analysis of population health data. Lancet. 2009;374(9693):881-92

10. Instituto Brasileiro de Geografia e Estatística (IBGE). Censo 2010. Disponível em: http:// cens02010.ibge.gov.br/. Acesso em: 29 mar. 2019.

11. Organização Mundial da Saúde (OMS). CID-10 - Classificação Estatística Internacional de Doenças vol. 1. 2008. Disponível em: http://www.datasus.gov.br/cid10/V2008/cid10.htm. Acesso em: 29 mar. 2019

12. Waiselfisz JJ. Mapa da violência 2015. Adolescentes de 16 e 17 anos do Brasil. 2015. Disponível em: https://www.mapadaviolencia.org.br/pdf2015/mapaViolencia2015_adolescentes.pdf. Acesso em: 29 mar. 2019.

13. Abasse MLF, de Oliveira RC, Silva TC, de Souza ER. Análise epidemiológica da morbimortalidade por suicídio entre adolescentes em Minas Gerais, Brasil. Ciênc Saúde Coletiva. 2009:14(2):407-16

14. Borges VR, Werlang BSG, Copatti M. Ideação suicida em adolescentes de 13 a 17 anos. Barbarói. 2008;7(28):109-23.

15. Ministério da Saúde. Secretaria de Vigilância em Saúde. Perfil epidemiológico das tentativas e óbitos por suicídio no Brasil e a rede de atenção à saúde. Bolettim Epidemiológico. 2017;48(30)

16. World Health Organization (WHO). Mental Health. Suicide Data. 2016. Disponível em: http://www.who.int/mental_health/prevention/suicide/suicideprevent/en/. Acesso em: 29 mar. 2019.

17. Machado DB, Santos DN. Suicídio no Brasil, de 2000 a 2012. J Bras Psiquiatr. 2015;64(1): 45-54.

18. Hesler LZ. Suicídio em municípios do sul do Brasil : um enfoque de gênero [dissertação]. Porto Alegre: Universidade do Rio Grande do Sul; 2013

19. Malta DC, Minayo MCS, Soares Filho AM, Silva MMA, Montenegro MMS, Ladeira RM, et al. Mortalidade e anos de vida perdidos por violências interpessoais e autoprovocadas no Brasil e Estados: análise das estimativas do Estudo Carga Global de Doença, 1990 e 2015. Rev Bras Epidemiol. 2017;20(Suppl 1):142-56.

20. Batista NO, Araújo JRC, Figueiredo PHM. Incidência e perfil epidemiológico de suicídios em crianças e adolescentes ocorridos no estado do Pará, Brasil, no período de 2010 a 2013. Rev Pan-Amazônica Saúde. 2016;7(4).

21. Pinto LW, Pires T0, Silva CMFP, Assis SG. Evolução temporal da mortalidade por suicídio em pessoas com 60 anos ou mais nos estados brasileiros, 1980 a 2009. Ciênc Saúde Coletiva. 2012;17(8):1973-81.

22. Wanzinack C, Temoteo A, Oliveira AL. Mortalidade por suicídio entre adolescentes/jovens brasileiros: um estudo com dados secundários entre os anos de 2011 a 2015. Divers Rev Eletrônica Interdiscip. 2017:10:106-17.

23. Kõlves K, De Leo D. Suicide rates in children aged 10-14 years worldwide: changes in the past two decades. Br J Psychiatry. 2014;205(4):283-5.

24. Souza LDDM, Silva RA, Jansen K, Kuhn RP, Horta BL, Pinheiro RT. Suicidal ideation in adolescents aged 11 to 15 years: Prevalence and associated factors. Rev Bras Psiquiatr. 2010;32(1):37-41

25. Silva LLT, Alvim CGG, Costa CC, Ramos TM, Costa EE. O suicídio na adolescência nas publicações da enfermagem brasileira: revisão integrativa da literatura. Rev Enferm Centro-0este Mineiro. 2016;5(3):1871-84.

26. Silva JL. Suicídios invisibilizados: investigação dos óbitos de adolescentes com intencionalidade indeterminada. São Paulo: Universidade de São Paulo; 2017.

27. Rede Interagencial de Informação para a Saúde. Indicadores básicos para a saúde no Brasil: conceitos e aplicações. Organ Pan-Americana da Saúde. 2008. Disponível em: http://tabnet.datasus.gov.br/tabdata/livroidb/2ed/indicadores.pdf. Acesso em: 29 mar. 2019.

28. Brasil. Ministério da Saúde. Portaria nº 1.876, de 14 de agosto de 2006. Institui Diretrizes Nacionais para Prevenção do Suicídio, a ser implantadas em todas as unidades federadas, respeitadas as competências das três esferas de gestão. 
Mortalidade por suicídio de adolescentes no Brasil: tendência temporal de crescimento entre 2000 e 2015

Suicide mortality among adolescents in Brazil: increasing time trend between 2000 and 2015 J Bras Psiquiatr. 2019;68(1):1-7

Where you read:

DOl: 10.1590/0047-2085000000218

Should read:

DOl: 10.1590/0047-2085000000345 